\title{
Change in serum KL-6 level from baseline is useful for predicting life-threatening EGFR-TKls induced interstitial lung disease
}

\author{
Shigeo Kawase ${ }^{1}$, Noboru Hattori ${ }^{1 *}$, Nobuhisa Ishikawa', Yasushi Horimasu', Kazunori Fujitaka', Osamu Furonaka ${ }^{2}$,
} Takeshi Isobe ${ }^{3}$, Seigo Miyoshi ${ }^{4}$, Hironobu Hamada ${ }^{4,5}$, Takashi Yamane ${ }^{6}$, Akihito Yokoyama ${ }^{6}$ and Nobuoki Kohno ${ }^{1}$

\begin{abstract}
Background: A high incidence of interstitial lung disease (ILD) has been reported in patients with advanced nonsmall cell lung cancer (NSCLC) treated with epidermal growth factor receptor-tyrosine kinase inhibitors (EGFR-TKIs), particularly in Japanese populations. A previous report from our laboratory demonstrated that KL-6 was a useful serum biomarker to assess the severity of drug-induced pneumonitis. Based on these observations, this study was conducted to evaluate the risk factors of EGFR-TKIs induced ILD and the usefulness of monitoring serum KL-6 levels in patients who developed EGFR-TKIs induced ILD in a large multi-institutional setting.
\end{abstract}

Methods: We retrospectively reviewed clinical records and radiographies of 341 patients with advanced NSCLCs who were treated with EGFR-TKIs, and analyzed risk factors for the development of EGFR-TKIs induced ILD. Changes of circulating levels of KL- 6 were also evaluated in the patients who developed EGFR-TKIs induced ILD.

Results: Among the 341 patients included in this study, 20 (5.9\%) developed EGFR-TKIs induced ILD, and 9 (2.6\%) died from ILD. Univariate analyses revealed that only preexisting pulmonary fibrosis was a significant risk factor for the development of EGFR-TKIs induced ILD $(p=0.003)$. Absolute levels of circulating KL-6 at neither baseline nor the onset of ILD could discriminate between life-threatening and non-life threatening EGFR-TKIs induced ILDs. However, we found that the ratios of serum KL-6 levels just after the onset of EGFR-TKIs induced ILD to those at baseline could quite precisely distinguish survivors from non-survivors $(p=0.006)$ as well as acute interstitial pneumonia (AIP) pattern from non-AIP pattern $(p=0.005)$.

Conclusions: The results of this study strongly support the potential of KL-6 as a diagnostic biomarker for lifethreatening EGFR-TKIs induced ILD. Monitoring of KL-6 is also useful to evaluate the progression and severity of EGFR-TKIs induced ILD.

Keywords: Lung cancer, KL-6, EGFR-TKI, interstitial lung disease

\section{Background}

Gefitinib (ZD1839, Iressa; AstraZeneca) and erlotinib (Tarceva, OSI-774; OSI Pharmaceuticals) are orally active epidermal growth factor receptor tyrosine kinase inhibitors (EGFR-TKIs) used for the treatment of nonsmall cell lung cancer (NSCLC) patients [1]. EGFR-TKIs sometimes cause drastic tumor regression in specific

\footnotetext{
* Correspondence: nhattori@hiroshima-u.ac.jp

'Department of Molecular and Internal Medicine, Graduate School of Biomedical Sciences, Hiroshima University, 1-2-3 Kasumi, Minami-ku, Hiroshima 734-8551, Japan

Full list of author information is available at the end of the article
}

subgroups of patients with advanced NSCLC, including women, non-smokers, patients with lung adenocarcinoma (ADC) histology, patients of Asian origin and patients with EGFR mutations [2-6]. On the other hand, treatment with EGFR-TKIs is associated with serious side effects, such as life-threatening drug-induced interstitial lung disease (ILD), particularly in Japanese populations [7-13]. These previous studies have reported that male gender, smoking history, poor performance status (PS), and preexisting ILD are risk factors for developing EGFR-TKIs induced ILD, however, we questioned whether each of these should be equally considered for 
the risk-benefit assessment to use EGFR-TKIs for the treatment of NSCLCs in a practical clinical setting. In addition, we also wondered whether we can assess the severity of EGFR-TKIs induced ILD when it develops during EGFR-TKIs treatment.

KL-6 is a mucin-like glycoprotein with a molecular weight of $200 \mathrm{kd}$ and has been classified as human MUC1 mucin [14-17]. Previous studies have demonstrated that serum levels of KL-6 are elevated in a variety of ILDs, such as idiopathic pulmonary fibrosis (IPF), collagen vascular disease associated interstitial pneumonitis, radiation pneumonitis, pulmonary sarcoidosis [18-26]. Furthermore, our laboratory has also demonstrated that absolute levels of KL-6 at the onset of druginduced ILD can predict the clinical outcomes [27]. Although our previous studies have suggested the usefulness of KL-6 as a tumor marker [28,29] and a predictor of survival in NSCLC patients treated with EGFRTKIs [30], significance of circulating KL-6 level as a detector of EGFR-TKIs induced ILD or a predictor of clinical outcome in patients with EGFR-TKIs induced ILD has not been determined yet.

In the cohort of the present study, to obtain more information on risk factors for developing EGFR-TKIs induced ILD, the characteristics of NSCLC patients who developed ILD during EGFR-TKIs treatment were analyzed. In addition, to evaluate whether monitoring serum KL-6 levels in NSCLC patients during the treatment is useful to detect the development of EGFR-TKIs induced ILD or predict the clinical outcome of EGFRTKIs induced ILD, circulating KL-6 levels were measured in NSCLC patients included in the cohort before and during EGFR-TKIs treatment.

\section{Methods}

\section{Study subjects}

Between August 2002 and August 2010, 341 advanced NSCLC patients treated with gefitinib (250 mg/day) or erlotinib (150 mg/day) at Hiroshima University Hospital (Hiroshima, Japan), Ehime University Hospital (Ehime, Japan), Shimane University Hospital (Shimane, Japan), Kochi University Hospital (Kochi, Japan) and Onomichi General Hospital (Hiroshima, Japan) were consecutively enrolled in the study. The disease staging was carried out using computed tomography (CT) scan of the chest and abdomen, bone scintigraphy or F-18 fluorodeoxyglucose positron emission tomography (FDG-PET/CT), and magnetic resonance imaging (MRI) of the head. To obtain information on both the response of tumor to EGFR-TKIs treatment and the occurrence of EGFRTKIs induced ILD, chest radiography and/or CT scans were performed at least once a month at each institution, and the patients were followed-up until 12 weeks after the administration of EGFR-TKIs. Informed consent was obtained from all patients. This study complied with the Declaration of Helsinki, and was approved by the individual institutional Ethical Committees.

\section{Diagnosis of preexisting pulmonary disorder and EGFR- TKIs induced ILD}

The presence of preexisting pulmonary fibrosis was determined according to the diagnostic criteria set by the ATS/ERS on the basis of clinical characteristic and/ or chest CT findings, and the types of preexisting pulmonary fibrosis were classified into idiopathic pulmonary fibrosis (IPF) pattern and non-IPF pattern [31-33]. In addition, the presence of preexisting pulmonary emphysema was determined by chest CT findings that show low attenuation areas occupying more than $25 \%$ of the entire lung field in at least one slice [34]. The diagnosis of EGFR-TKIs induced ILD was made using the diagnostic algorithm described elsewhere [11,35]. We defined EGFR-TKIs induced ILD as diffuse pulmonary infiltrates newly developed during EGFR-TKIs treatment with lack of evidence for alternative diseases such as infection, tumor progression, heart failure and pulmonary embolism. When the occurrence of EGFR-TKIs induced ILDs was suspected, chest CT scans were performed, levels of brain natriuretic peptide (BNP) and Ddimer in blood were measured, the sputum culture, blood culture, urine antigen test for Legionella pneumophila and Streptococcus pneumoniae, cytomegalovirus antigen test, and polymerase chain reaction test for Pneumocystis jiroveci were conducted. When possible, bronchoalveolar lavage or lung biopsy was carried out. Tumor progression was carefully excluded on the basis of the clinical information including chest CT findings, physical examinations, and tumor markers. The final diagnosis of EGFR-TKIs induced ILD was made by the consensus of at least two independent pulmonologists. We collected the clinical information of all 341 patients, such as patient age, sex, histologic type, disease stage, performance status, prior chemotherapy and thoracic radiation therapy, preexisting pulmonary fibrosis, preexisting pulmonary emphysema, EGFR mutation status, types of EGFR-TKIs, duration of EGFR-TKIs treatment and laboratory data.

\section{Subclassification of EGFR-TKIs induced ILD}

The chest radiography and $\mathrm{CT}$ of the patients who developed EGFR-TKIs induced ILD were reviewed separately by two independent observers who were not aware of the patients' profiles, and were categorized into four patterns as previously described [27,36]: (1) acute interstitial pneumonia (AIP) pattern characterized by extensive bilateral ground glass attenuation or airspace consolidations with traction bronchiectasis, (2) chronic interstitial pneumonia (CIP) pattern characterized by 
fibrosis and/or consolidation, (3) cryptogenic organizing pneumonia/eosinophilic pneumonia (COP/EP) pattern showing peribronchial or subpleural consolidation without fibrosis, and (4) hypersensitivity pneumonitis (HP) pattern with diffuse ground glass opacities without fibrosis.

\section{EGFR mutation status}

In 148 out of 341 NSCLC patients included in the study, EGFR mutation statuses were assessed using paraffinembedded biopsy samples or surgically resected tumor tissues. To evaluate EGFR mutations, the peptide nucleic acid-locked nucleic acid polymerase chain reaction (PNA-LNA PCR) clamp test that can detect G719C, G719S, G719A, L858R, L861Q, T790M and 7 different exon 19 deletions [37] was used.

\section{Electrochemiluminescence immunoassay (ECLIA) to determine circulating levels of KL- 6}

At least one serum sample was obtained before the EGFRTKIs treatment from each patient included in the study. From 15 out of 20 patients who developed EGFR-TKIs induced ILD, a total of 2-5 serum samples per patient were also collected weekly after the occurrence of EGFRTKIs induced ILD, and stored at $-80^{\circ} \mathrm{C}$. Serum KL- 6 levels were measured by sandwich-type electrochemiluminescence immunoassay (ECLIA) using a Picolumi 8220 Analyzer (Eidia, Tokyo, Japan), as previously described $[29,30]$.

\section{Statistical analysis}

The data were analyzed with a statistical software package (JMP, version 7.0.1; SAS Institute Inc.; Cary, North Carolina) and $p<0.05$ indicated a significant difference. Data are shown as the mean \pm SEM. Differences between patients with and without preexisting pulmonary fibrosis, survivors and non-survivors, and patients with AIP pattern and the other patterns of EGFR-TKIs induced ILD were analyzed using the Mann-Whitney U-test. We analyzed differences between patients with preexisting pulmonary fibrosis who developed EGFR-TKIs induced ILD or not using the Fisher's exact test. In order to test differences among the variables evaluated prior to and at the diagnosis of EGFR-TKIs induced ILD, Wilcoxon test was used. The risk factors associated with EGFR-TKIs induced ILD were evaluated using multiple logistic regression analysis. The criterion for removing a variable was the likelihood ratio statistic, which was based on the maximum partial likelihood estimate (default $p$-value of 0.05 for removal from the model).

\section{Results}

\section{Characteristics of patients}

Table 1 shows the characteristics of the 341 patients enrolled in this study. All patients were Japanese. The
Table 1 Patients' characteristics of 341 patients treated with EGFR-TKIs

\begin{tabular}{|c|c|c|}
\hline Characteristics & No. of patients & $\%$ patients \\
\hline Total & 341 & 100 \\
\hline \multicolumn{3}{|l|}{ Age (years) } \\
\hline Mean $( \pm$ SEM) & $65.2( \pm 0.6)$ & \\
\hline$<60$ & 102 & 29.9 \\
\hline$\geq 60$ & 239 & 70.1 \\
\hline \multicolumn{3}{|l|}{ Sex } \\
\hline Female & 167 & 49.0 \\
\hline Male & 174 & 51.0 \\
\hline \multicolumn{3}{|l|}{ Histologic type } \\
\hline Adenocarcinoma & 296 & 86.8 \\
\hline Squamous cell carcinoma & 34 & 10.0 \\
\hline Others & 11 & 3.2 \\
\hline \multicolumn{3}{|l|}{ Smoking history } \\
\hline Current & 60 & 17.6 \\
\hline Former & 110 & 32.3 \\
\hline Never & 171 & 50.1 \\
\hline \multicolumn{3}{|l|}{ Disease stage } \\
\hline IV & 206 & 60.4 \\
\hline$\| \mathrm{IIB}$ & 54 & 15.8 \\
\hline$|-||| A$ & 18 & 5.3 \\
\hline Recurrence after surgery & 63 & 18.5 \\
\hline \multicolumn{3}{|l|}{ Performance status } \\
\hline$\geq 2$ & 141 & 41.3 \\
\hline $0-1$ & 200 & 58.7 \\
\hline \multicolumn{3}{|c|}{ No. of prior chemotherapy regimens } \\
\hline$\geq 2$ & 118 & 34.6 \\
\hline $0-1$ & 223 & 65.4 \\
\hline \multicolumn{3}{|l|}{ Prior thoracic radiotherapy } \\
\hline Yes & 47 & 13.8 \\
\hline No & 294 & 86.2 \\
\hline \multicolumn{3}{|l|}{ Preexisting pulmonary fibrosis } \\
\hline Yes & 48 & 14.1 \\
\hline No & 293 & 85.9 \\
\hline \multicolumn{3}{|c|}{ Preexisting pulmonary emphysema } \\
\hline Yes & 82 & 24.0 \\
\hline No & 259 & 76.0 \\
\hline \multicolumn{3}{|l|}{ EGFR mutation status } \\
\hline Wild type & 57 & 16.7 \\
\hline Mutant & 91 & 26.4 \\
\hline Not evaluated & 193 & 56.9 \\
\hline \multicolumn{3}{|l|}{ Types of EGFR-TKI } \\
\hline Gefitinib & 302 & 88.6 \\
\hline Erlotinib & 39 & 11.4 \\
\hline
\end{tabular}

ages of the patients ranged from 30 to 87 years (mean age $65.2 \pm 0.6 \mathrm{SEM})$. Of the patients, 167 (49.0\%) were female, 296 (86.8\%) had adenocarcinomas (ADCs), 171 (50.1\%) were never smokers, and $200(58.7 \%)$ were in good performance status ( $\mathrm{PS}=0,1)$. Forty-seven (13.8\%) patients received thoracic radiations prior to 
EGFR-TKIs treatment, and preexisting ILDs were identified in $48(14.1 \%)$ patients. Twenty-six $(55.3 \%)$ out of the 47 patients who underwent radiation therapy had preexisting pulmonary fibrosis. Preexisting pulmonary emphysema was identified in $82(24.0 \%)$ patients. PNALNA-PCR clamp tests to detect EGFR mutations could be performed in 148 (43.4\%) patients, and in 91 patients, EGFR mutations were detected: L858R mutation in 38 patients, G719S mutation in 2 patients, exon 19 deletions in 45 patients, and other types of mutations in 6 patients. Figure 1 shows the absolute serum KL-6 levels at the baseline according to the presence of preexisting pulmonary fibrosis. The absolute serum KL-6 levels at the baseline showed no significant difference between patients with and without preexisting pulmonary fibrosis (Mann-Whitney U-test; $p=0.207$ ). Table 2 shows the characteristics of the 48 patients who had preexisting pulmonary fibrosis. Eight (16.7\%) out of the 48 patients with preexisting pulmonary fibrosis developed EGFRTKIs induced ILD. Statistical analyses were made to see the association between the patients' characteristic and the development of EGFR-TKIs induced ILD among these patients (Table 2). In the patients who had preexisting pulmonary fibrosis, thoracic radiation prior to EGFR-TKIs treatment was not associated with the development of EGFR-TKIs induced ILD, however, there was a weak but statistically significant association between the development of EGFR-TKIs induced ILD and EGFR mutation status $(p=0.0498)$.

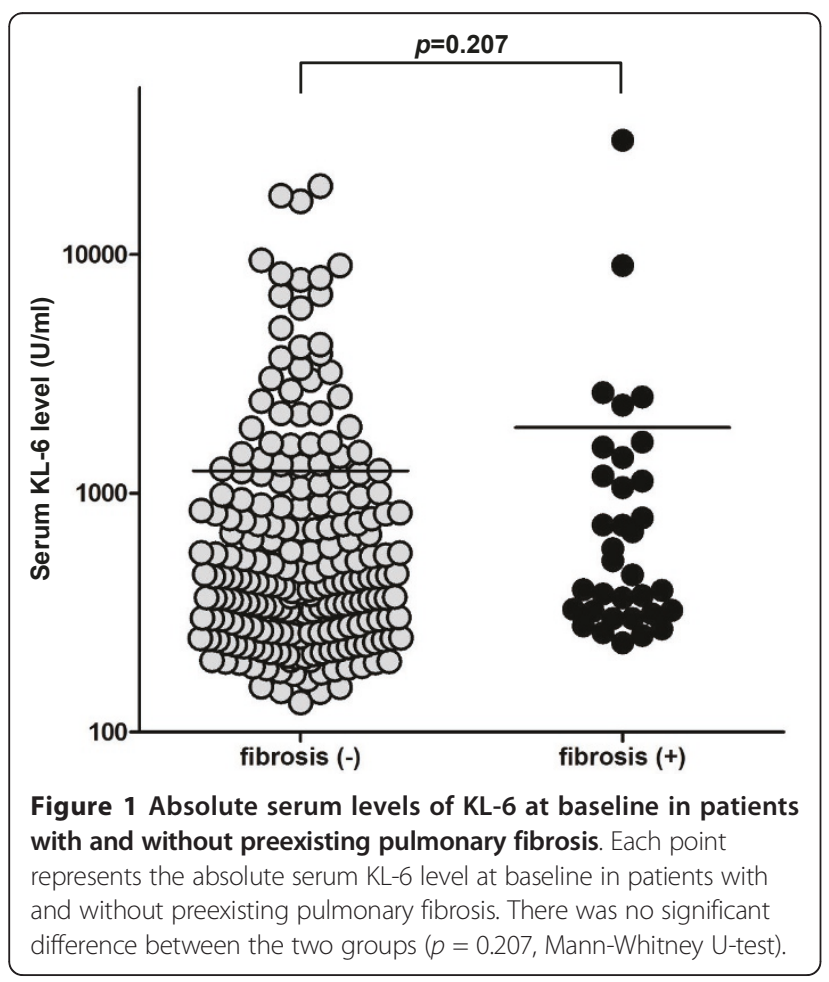

Incidence and characteristics of patients with EGFR-TKIs induced ILD

Among the 341 patients included in this study, 20 (5.9\%) developed EGFR-TKIs induced ILD, and 9 (2.6\%) died from ILD. Table 3 shows the characteristics and clinical course of these 20 patients. All the patients had acute onset or exacerbation of respiratory symptoms. The median interval from the administration of EGFRTKI to the occurrence of EGFR-TKIs induced ILD was 19 days (range 5-51 days). The subclassifications of EGFR-TKIs induced ILD categorized by the findings of chest CT scans in these 20 patients were as follows: AIP pattern in 5 patients, COP/EP pattern in 9 patients, and HP pattern in 6 patients. The CT images of 5 patients who demonstrated AIP pattern are shown in Figure 2. When the occurrence of EGFR-TKIs induced ILD was suspected, the administration of EGFR-TKI was immediately stopped and high dose methylprednisolone (1,000 mg daily for 3 days) therapy was started. All of the 5 patients with AIP patterns were refractory to the treatment and eventually died, whereas 7 of 9 patients with COP/EP pattern and 4 of 6 patients with HP pattern showed immediate response to the treatment. Postmortem examinations were performed in 3 patients (patient No. 5, 8 and 11) and diffuse alveolar damage (DAD) was detected histologically in all of them. In addition, the presence of preexisting pulmonary fibrosis was suspected in 2 of the 3 patients. Neither infection nor lymphangitic spread of cancer cells was pointed out in any of them.

\section{Risk factors for developing EGFR-TKIs induced ILD}

The results of univariate analyses on risk factors for EGFR-TKIs induced ILD are shown in Table 4. Univariate analyses revealed that only preexisting pulmonary fibrosis (odds ratio, 4.683; 95\% CI, 1.741-12.042; $p=$ 0.003 ) was a significant risk factor for the development of EGFR-TKIs induced ILD.

\section{Serum levels of KL-6 in patients who developed EGFR-} TKIs induced ILD

After the administration of the EGFR-TKIs, measurements of serum KL- 6 levels at least once during and/or around 4 weeks were achieved in 15 out of 20 patients who developed EGFR-TKIs induced ILD and 198 out of 321 patients who did not. The ratios of serum KL-6 levels during or around 4 weeks after the start of EGFRTKIs to those at baseline were $1.315 \pm 0.120$ for the former and $1.000 \pm 0.036$ for the latter, respectively (mean \pm SEM). There was a significant statistical difference between these ratios ( $p=0.004$, Mann-Whitney U-test). Figure 3 shows the serum levels of KL-6 at the multiple time points before and after the onset of ILD in 8 survivors (Figure 3A) and 7 non-survivors (Figure 3B). The 
Table 2 Patients' Characteristics of $\mathbf{4 8}$ patients with preexisting pulmonary fibrosis

\begin{tabular}{|c|c|c|c|c|}
\hline Characteristics & Total & EGFR-TKIs induced ILD (+) & EGFR-TKIs induced ILD (-) & $\mathrm{p}$-value \\
\hline Total & 48 & 8 & 40 & \\
\hline \multicolumn{5}{|l|}{ Age (years) } \\
\hline Mean $( \pm$ SEM) & & $67.5( \pm 3.6)$ & $66.2( \pm 1.8)$ & \\
\hline$<60$ & 12 & 2 & 10 & 1.000 \\
\hline$\geq 60$ & 36 & 6 & 30 & \\
\hline \multicolumn{5}{|l|}{ Sex } \\
\hline Female & 11 & 3 & 8 & 0.361 \\
\hline Male & 37 & 5 & 32 & \\
\hline \multicolumn{5}{|l|}{ Histologic type } \\
\hline Adenocarcinoma & 37 & 5 & 32 & 0.361 \\
\hline Squamous cell carcinoma/Others & 11 & 3 & 8 & \\
\hline \multicolumn{5}{|l|}{ Smoking history } \\
\hline Current/Former & 40 & 5 & 35 & 0.116 \\
\hline Never & 8 & 3 & 5 & \\
\hline \multicolumn{5}{|l|}{ Disease stage } \\
\hline IV & 24 & 3 & 21 & 0.701 \\
\hline |-I||B/Recurrence after surgery & 11 & 5 & 19 & \\
\hline \multicolumn{5}{|l|}{ Performance status } \\
\hline$\geq 2$ & 26 & 5 & 21 & 0.710 \\
\hline $0-1$ & 22 & 3 & 19 & \\
\hline \multicolumn{5}{|l|}{ No. of prior chemotherapy regimens } \\
\hline$\geq 2$ & 20 & 1 & 19 & 0.116 \\
\hline $0-1$ & 28 & 7 & 21 & \\
\hline \multicolumn{5}{|l|}{ Prior thoracic radiotherapy } \\
\hline Yes & 10 & 0 & 10 & 0.177 \\
\hline No & 38 & 8 & 30 & \\
\hline \multicolumn{5}{|l|}{ Pattern of preexisting pulmonary fibrosis } \\
\hline IPF pattern & 3 & 1 & 2 & 0.429 \\
\hline Non-IPF pattern & 45 & 7 & 38 & \\
\hline \multicolumn{5}{|l|}{ Preexisting pulmonary emphysema } \\
\hline Yes & 24 & 3 & 21 & 0.701 \\
\hline No & 24 & 5 & 19 & \\
\hline \multicolumn{5}{|l|}{ EGFR mutation status } \\
\hline Wild type & 9 & 5 & 4 & $0.0498^{*}$ \\
\hline Mutant & 11 & 1 & 10 & \\
\hline (Not evaluated) & (28) & (2) & (26) & \\
\hline \multicolumn{5}{|l|}{ Types of EGFR-TKI } \\
\hline Gefitinib & 40 & 5 & 35 & 0.116 \\
\hline Erlotinib & 8 & 3 & 5 & \\
\hline
\end{tabular}

${ }^{*} p<0.05$ (Fisher's exact test)

serum levels of KL-6 in 7 non-survivors but not in 8 survivors showed consistent trends to increase after the onset of EGFR-TKIs induced ILD. The absolute serum KL-6 levels at the onset as well as at baseline showed no difference between the 7 non-survivors and 8 survivors (Mann-Whitney $\mathrm{U}$-test; $p=0.072$ at onset, and $p=$ 0.072 at baseline, respectively). To assess the changes in serum KL- 6 level before and after the onset of ILD, the ratio of serum KL-6 level just after the onset of ILD to that at baseline was calculated in 15 of 20 patients who developed ILD. The differences in the ratios of serum KL-6 levels just after the onset of ILD from baseline were found to be statistically significant between the survivors and non-survivors (Mann-Whitney U-test; $p=$ 0.006; Figure 4).

Then, we compared the circulating levels of KL- 6 according to the patterns of EGFR-TKIs induced ILD subclassified by the manifestation on chest CT in 15 of 20 patients who developed EGFR-TKIs induced ILD. The absolute levels of circulating KL-6 at neither baseline nor 
Table 3 Characteristics of 20 patients with EGFR-TKIs induced ILD

\begin{tabular}{|c|c|c|c|c|c|c|c|c|c|c|c|c|c|c|c|}
\hline No & Age & Sex & $\begin{array}{l}\text { Histological } \\
\text { type }\end{array}$ & $\begin{array}{l}\text { Smoking } \\
\text { history }\end{array}$ & Stage & PS & $\begin{array}{l}\text { Prior } \\
\text { CT }\end{array}$ & $\begin{array}{c}\text { Prior } \\
\text { RT }\end{array}$ & $\begin{array}{l}\text { Preexisting } \\
\text { fibrosis }\end{array}$ & $\begin{array}{c}\text { Preexisting } \\
\text { emphysema }\end{array}$ & $\begin{array}{c}E G F R \\
\text { mutation }\end{array}$ & $\begin{array}{c}\text { Type of EGFR- } \\
\text { TKI }\end{array}$ & $\begin{array}{c}\text { Length of EGFR- } \\
\text { TKI }\end{array}$ & $\begin{array}{c}\mathrm{CT} \\
\text { findings }\end{array}$ & Prognosis \\
\hline 1 & 68 & $M$ & SCC & Ex & IIIB & 1 & 1 & No & No & Yes & N.E. & Gefitinib & 11 & COP/EP & Alive \\
\hline 2 & 80 & M & SCC & Never & $\operatorname{Rec}$ & 2 & 1 & No & Yes & Yes & Wild & Gefitinib & 17 & COP/EP & Alive \\
\hline 3 & 70 & $\mathrm{~F}$ & SCC & Never & $\| I \mid A$ & 1 & 1 & Yes & No & No & N.E. & Gefitinib & 24 & $\mathrm{HP}$ & Alive \\
\hline 4 & 60 & M & $A D C$ & Never & $\| \mathrm{B}$ & 1 & 1 & No & No & No & N.E. & Gefitinib & 35 & COP/EP & Alive \\
\hline 5 & 68 & $\mathrm{~F}$ & ADC & Ex & $\operatorname{Rec}$ & 1 & 2 & No & No & No & Wild & Gefitinib & 16 & AIP & Dead \\
\hline 6 & 60 & M & $A D C$ & Current & $\| \mathrm{B}$ & 1 & 2 & No & No & Yes & N.E. & Gefitinib & 26 & COP/EP & Alive \\
\hline 7 & 57 & M & $A D C$ & Never & $\operatorname{Rec}$ & 0 & 3 & No & No & No & L858R & Gefitinib & 51 & COP/EP & Alive \\
\hline 8 & 73 & M & $A D C$ & Current & IV & 4 & 0 & No & Yes & Yes & N.E. & Gefitinib & 13 & AlP & Dead \\
\hline 9 & 65 & $\mathrm{~F}$ & $A D C$ & Never & IV & 2 & 3 & No & No & No & N.E. & Gefitinib & 38 & $\mathrm{HP}$ & Dead \\
\hline 10 & 69 & $\mathrm{~F}$ & $A D C$ & Never & $\||| B$ & 2 & 1 & No & Yes & No & N.E. & Gefitinib & 14 & AIP & Dead \\
\hline 11 & 84 & $\mathrm{~F}$ & $A D C$ & Ex & $\| \mathrm{B}$ & 4 & 0 & No & Yes & No & Wild & Gefitinib & 16 & AIP & Dead \\
\hline 12 & 63 & $\mathrm{~F}$ & SCC & Never & IV & 1 & 1 & No & Yes & No & N.E. & Gefitinib & 50 & COP/EP & Dead \\
\hline 13 & 67 & $\mathrm{~F}$ & $A D C$ & Never & $\operatorname{Rec}$ & 0 & 1 & No & No & No & Deletion & Gefitinib & 48 & $\mathrm{HP}$ & Alive \\
\hline 14 & 60 & M & $A D C$ & Current & IV & 4 & 0 & No & Yes & Yes & L858R & Gefitinib & 17 & $\mathrm{HP}$ & Dead \\
\hline 15 & 55 & M & $A D C$ & Ex & IV & 3 & 4 & No & No & No & L858R & Gefitinib & 47 & COP/EP & Dead \\
\hline 16 & 69 & M & $A D C$ & Current & IV & 3 & 1 & No & Yes & No & Wild & Erlotinib & 14 & AIP & Dead \\
\hline 17 & 56 & M & SCC & Current & $\operatorname{Rec}$ & 0 & 1 & No & Yes & Yes & Wild & Erlotinib & 21 & COP/EP & Alive \\
\hline 18 & 59 & M & ADC & Ex & $\operatorname{Rec}$ & 1 & 2 & No & Yes & No & Wild & Erlotinib & 5 & $\mathrm{HP}$ & Alive \\
\hline 19 & 66 & M & $A D C$ & Current & $\| \mathrm{BB}$ & 0 & 0 & No & No & No & L858R & Gefitinib & 17 & COP/EP & Alive \\
\hline 20 & 64 & $\mathrm{~F}$ & $A D C$ & Never & $\operatorname{Rec}$ & 1 & 1 & No & No & No & L858R & Erlotinib & 31 & HP & Alive \\
\hline
\end{tabular}

Abbreviations: ADC, Adenocarcinoma; SCC, Squamous cell carcinoma; Rec, Recurrence after the surgery; CT, Chemotherapy; RT, Radiation therapy; N.E, Not evaluated; COP/EP, Cryptogenic organizing pneumonia/ Eosinophilic pneumonia; HP, Hypersensitivity pneumonitis; DAD, Diffuse alveolar damage. 


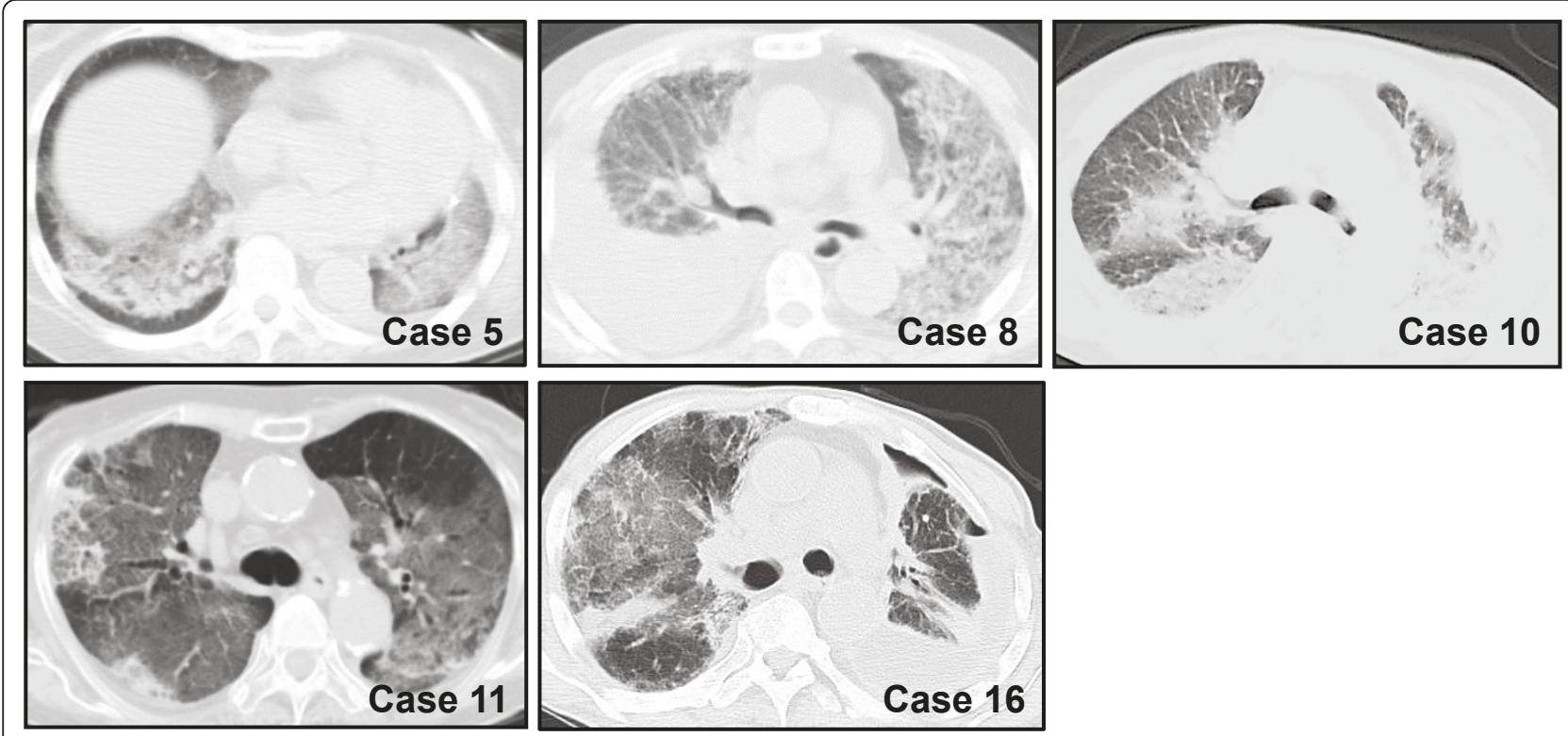

Figure 2 Chest CT images of five patients who developed EGFR-TKI induced acute interstitial pneumonia (AIP). Representative chest CT images of the five patients who developed AIP pattern of EGFR-TKIs induced ILD are shown. Each case number corresponds to the patient's number listed in Table 3.

the onset of ILD were found not to be statistically significant between the life-threatening pattern (AIP pattern) of 4 patients and the other patterns of 11 patients (MannWhitney U-test; $p=0.648$ at onset, and $p=0.845$ at baseline, respectively). When the ratio of serum KL-6 level at baseline to that at the onset of ILD was compared, this value was significantly higher in the patients with the lifethreatening pattern (AIP pattern) than that in other patterns (Mann-Whitney U-test; $p=0.005$; Figure 5). In addition, patients whose serum KL-6 levels rose more than 1.5 times higher than their baseline levels had a high chance of developing the AIP pattern.

\section{Discussion}

In this large multi-institutional study, we investigated the incidence and risk factors for developing ILD in patients treated with EGFR-TKIs until 12 weeks after the start of EGFR-TKIs therapy. Univariate analyses revealed that preexisting pulmonary fibrosis at baseline was the only risk factor for EGFR-TKIs induced ILD. Although absolute serum KL-6 levels at neither baseline nor the onset of ILD could discriminate between lifethreatening and non-life-threatening EGFR-TKIs induced ILDs, the ratio of serum KL-6 level at the occurrence of EGFR-TKIs induced ILD to that at

Table 4 Risk factors for EGFR-TKIs induced ILD at the start of EGFR-TKIs

\begin{tabular}{|c|c|c|c|c|}
\hline Variables & & Odds ratio & $95 \% \mathrm{Cl}$ & $P$-value \\
\hline \multicolumn{5}{|l|}{ Univariate analysis } \\
\hline Age (years) & $\geq 60 /<60$ & 1.758 & $0.626-6.256$ & 0.301 \\
\hline Gender & Male/Female & 1.472 & $0.593-3.848$ & 0.406 \\
\hline Histological type & Non-ADC/ADC & 2.342 & $0.730-6.420$ & 0.142 \\
\hline Smoking history & Never/Smoker & 1.006 & $0.402-2.516$ & 0.989 \\
\hline Performance status & $\geq 2 / 0-1$ & 0.942 & $0.360-2.341$ & 0.899 \\
\hline No. of prior chemotherapy regimens & $\geq 2 / 0-1$ & 0.800 & $0.277-2.053$ & 0.652 \\
\hline Prior thoracic radiotherapy & Yes/No & 0.315 & $0.017-1.575$ & 0.187 \\
\hline Preexisting pulmonary fibrosis & Yes/No & 4.683 & $1.741-12.042$ & $0.003^{*}$ \\
\hline Preexisting pulmonary emphysema & Yes/No & 1.382 & $0.476-3.574$ & 0.531 \\
\hline EGFR mutation & Wild type/EGFR mutant & 1.667 & $0.497-5.594$ & 0.400 \\
\hline Types of EGFR-TKI & Gefitinib/Erlotinib & 2.043 & $0.562-5.948$ & 0.253 \\
\hline Serum KL-6 level at baseline $(\mathrm{U} / \mathrm{ml})$ & $\geq 500 /<500$ & 2.096 & $0.679-7.116$ & 0.199 \\
\hline
\end{tabular}

${ }^{*} \mathrm{P}<0.05$

Abbreviation: ADC, Adenocarcinoma. 



Figure 3 Kinetics of serum KL-6 levels in (A) 8 survivors and (B) 7 non-survivors who developed EGFR-TKIs induced ILD. Week 0 is designated as the week when EGFR-TKIs induced ILD was diagnosed. Before and after the onset of EGFR-TKIs induced ILD, the serum levels of $\mathrm{KL}-6$ showed a trend not to change in the survivors but to increase in the non-survivors.

baseline was found to quite precisely do so. These findings suggest the significance of serum KL-6 level for the detection of life threatening EGFR-TKIs induced ILD.

The development of molecular targeted agents has been a key factor in recent advances in cancer therapy, and some of these agents have been applied in clinical practice. EGFR-TKIs are one of the representative molecular target agents and, at first, were considered to be safe agents with mild side effects in comparison to cytotoxic agents. However, following the increase in usage of EGFR-TKIs in lung cancer therapy, a significantly higher

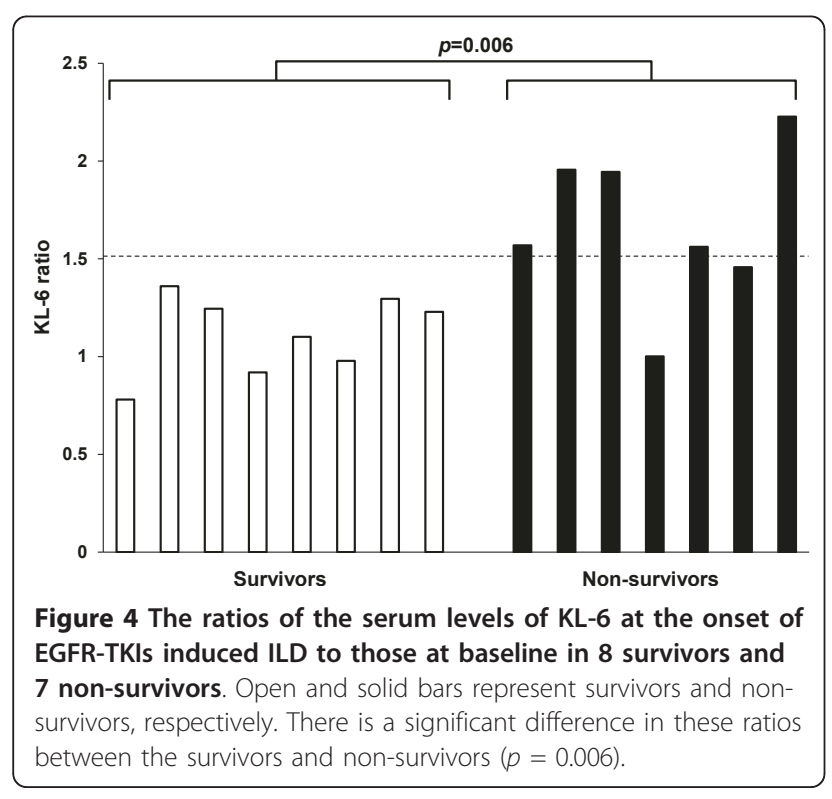

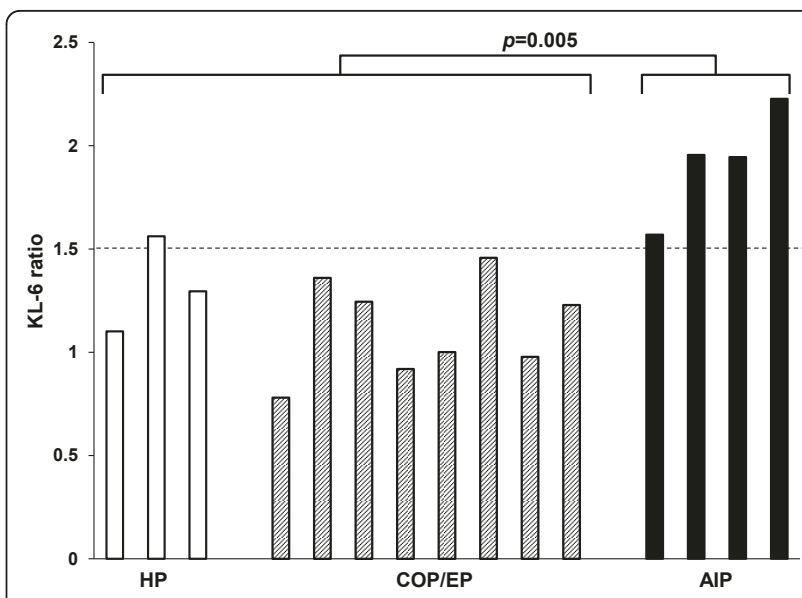

Figure 5 The ratios of the serum levels of $\mathrm{KL}-6$ at the onset of EGFR-TKI related ILD to those at baseline on the basis of the sub-classifications of EGFR-TKIs induced ILD. Open, shaded, and solid bars represent hypersensitivity pneumonitis (HP) pattern,

cryptogenic organizing pneumonia/eosinophilic pneumonia (COP/

EP) pattern, and acute interstitial pneumonia (AIP) pattern,

respectively. There is a significant difference in these ratios between AIP pattern and the other patterns ( $p=0.005$ ). incidence of life-threatening drug induced ILD in Japanese patients than that of patients in the rest of the world was reported [38,39]. In the present study, out of 341 NSCLC patients treated with EGFR-TKIs, 20 patients (5.9\%) developed ILD and 9 patients $(2.6 \%)$ died from ILD. The incidence and mortality of EGFR-TKIs induced ILD were relatively higher than those reported in previous studies from Japan [7-13,39]. This result might be due to the high incidence of preexisting pulmonary fibrosis in this study. In this study, the manifestations of chest CT scans in 20 patients who developed EGFR-TKIs induced ILD were classified as AIP pattern for 5 patients, COP/EP pattern for 9 patients and HP pattern for 6 patients. Interestingly, CIP pattern was not observed as was the case in a previous study [36]. All the patients who demonstrated the AIP pattern died, whereas the majority of patients with other patterns recovered from EGFR-TKIs induced ILD. In this study, the postmortem examination of three patients with AIP pattern revealed that DAD was the main cause of death and observations similar to ours have been reported previously $[7,8]$. In this study, univariate analysis revealed that preexisting pulmonary fibrosis was the only risk factor for developing EGFR-TKIs induced ILD. Although previous studies reported that male gender, smoking history and poor PS were also independent risk factors for developing EGFR-TKIs induced ILD [7-13,39], neither of them correlated with incidence or mortality of EGFR-TKIs induced ILD in the present study. This may be due to the small sample size and high incidence of preexisting pulmonary fibrosis in our studied patients. 
Although a previous study from our laboratory reported that serum KL-6 levels at diagnosis increased only in the life-threatening types, such as the DAD and CIP patterns, of drug induced ILDs [27], absolute serum KL-6 levels at the onset of EGFR-TKIs induced ILD did not correlate with clinical outcomes in the present study. The immunohistochemical analysis of KL-6 using three postmortem autopsy specimens showed that KL-6 was expressed at tumor cells in the primary lesions as well as alveolar epithelial cells in the EGFR-TKIs induced ILDs (data not shown). Therefore, we speculate that the origin of serum KL- 6 at the onset of EGFRTKIs induced ILD might be associated with both NSCLCs and EGFR-TKIs induced ILDs. On the other hand, we found that the ratios of serum KL-6 levels just after the onset of ILD to those at baseline could quite precisely discriminate life-threatening ILD from nonlife-threatening ILD, and correlate well with the disease progression. We can speculate that a drastic increase in serum KL-6 levels after the administration of EGFRTKIs might be due to severe lung injury accompanied with both alveolar-capillary destruction and enhancement of alveolar-capillary permeability which allow KL6 to leak into the circulation from the alveolar space [40]. Based on these observations, KL-6 can be regarded as a good serum biomarker to assess the severity of alveolar epithelium injury and the clinical outcome of EGFR-related ILD. Regarding the association between KL-6 and other serum biomarkers for ILD such as surfactant protein (SP)-A and SP-D in EGFR-TKIs induced ILD, we do not have data to discuss. Previous studies, which measured serum SP-A, SP-D, and KL-6 levels in 4 patients with EGFR-TKIs induced ILD, demonstrate that serum SP-A and SP-D levels increased in all studied patients whereas KL-6 levels only elevated in patients with life-threatening EGFR-TKIs induced ILD $[8,41]$. This observation is compatible with the findings of the present study.

In addition to its ability to detect patients who develop life-threatening ILD, the monitoring of serum KL-6 levels is also useful to predict survival and progressive disease in NSCLC patients treated with EGFR-TKIs [30]. As measurement of serum KL-6 level is more rapid, inexpensive, reproducible, and easier to perform than CT scans, its monitoring could be quite useful to assess the condition of NSCLC patients receiving EGFRTKIs. The development of EGFR-TKIs induced ILD is reported to mostly occur within the first 4 weeks after the start of EGFR-TKIs [11]. In the present study, 5 cases developed ILD within the first 2 weeks (ranged from 5 to 14 days) after the start of EGF-TKIs. Therefore, based on the results of the present study, once a week monitoring of serum KL-6 levels in addition to chest radiography could be recommended for NSCLC patients receiving EGFR-TKIs particularly for the first 4 weeks after the start of treatment.

Although these promising results were obtained, we are aware that this study has a number of limitations. First, the number of EGFR-TKIs induced ILD patients included in the study was not sufficient for a valid statistical analysis. Second, this study was conducted in a retrospective manner. Therefore, the information on EGFR mutation statuses in cancer tissue was not obtained from all the studied patients. Furthermore, multiple measurements of serum KL-6 levels were not achieved in all patients who developed EGFR-TKIs induced ILD. Third, the enrolled NSCLC patients might be biased compared with general advanced NSCLC population. We believe that this was caused by our trend to use EGFR-TKIs for specific subgroups of NSCLC patients such as women, non-smokers, and patients with EGFR mutations. Finally, the studied patients were only Japanese. Considering ethnic differences in the efficacy of EGFR-TKIs treatment and/or the occurrence of adverse side effects related by EGFRTKIs, we should carefully interpret the results when this monitoring system is applied to non-Japanese patients. A large and prospective study to measure serum KL-6 levels serially before and after EGFR-TKIs treatment, also including non-Japanese patients, will be required to evaluate the utility of monitoring KL-6 in EGFR-TKIs induced ILDs.

\section{Conclusions}

Our results indicate that the change in serum KL-6 level from baseline should be useful biomarker for the diagnosis of life-threatening EGFR-TKIs induced ILD and for estimating its progress and severity. A risk-benefit analysis and patient selection should be considered as well as close monitoring of serum levels of KL-6, particularly if using EGFR-TKIs in patients with preexisting pulmonary fibrosis.

\section{List of Abbreviations}

EGFR-TKI: epidermal growth factor receptor tyrosine kinase inhibitor; NSCLC: non-small cell lung cancer; ADC: adenocarcinoma; ILD: interstitial lung disease; KL-6: Krebs von den Lungen-6; IPF: idiopathic pulmonary fibrosis; AIP: acute interstitial pneumonia; CIP: chronic interstitial pneumonia; CT: computed tomography; FDG-PET: F-18 fluorodeoxyglucose positron emission tomography; MRI: magnetic resonance imaging; COP/EP: cryptogenic organizing pneumonia/eosinophilic pneumonia; HP: hypersensitivity pneumonitis; PNA-LNA PCR: peptide nucleic acid-locked nucleic acid polymerase chain reaction; ECLIA: electrochemiluminescence immunoassay.

\section{Acknowledgements}

This work was partly supported by Grants-in-Aid for Scientific Research from the Minister of Education, Culture, Sports, Science and Technology of Japan.

\section{Author details}

${ }^{1}$ Department of Molecular and Internal Medicine, Graduate School of Biomedical Sciences, Hiroshima University, 1-2-3 Kasumi, Minami-ku, Hiroshima 734-8551, Japan. ²Department of Respiratory Medicine, Onomichi 
General Hospital, 7-19 Kohama, Onomichi, Hiroshima 722-8508, Japan. ${ }^{3}$ Department of Clinical Oncology and Respiratory Medicine, Shimane University, 89-1, Enya-cho, Izumo, Shimane 693-8501, Japan. ${ }^{4}$ Department of Integrated Medicine and Informatics, Ehime University Graduate School of Medicine, Toon, Ehime 791-0295, Japan. ${ }^{5}$ Department of Health and Sports Medical Sciences, Graduate School of Health Sciences, Hiroshima University, Hiroshima, Japan. 'Department of Hematology and Respiratory Medicine, Kochi Medical School, Kochi University, Nankoku, Kochi 783-8505, Japan.

\section{Authors' contributions}

SK performed part of the statistical analysis and drafted the manuscript. $\mathrm{NH}$ conceived the study, and participated in its design and coordination and helped to draft the manuscript. NI conceived the study, and participated in patient recruitment and helped to draft the manuscript. $\mathrm{YH}$ performed part of the statistical analysis and participated in creating the figures. KF, OF, TI, $\mathrm{SM}, \mathrm{HH}$ and TY participated in the selection and collection of patient material. AY conceived the study, and participated in its design and coordination. NK conceived the study, and participated in its design and coordination and supervised the study. All authors read and approved the final manuscript.

\section{Competing interests}

Nobuoki Kohno has a personal royalty of KL-6 from a Japanese pharmaceutical company, Eisai Co., Ltd. The remaining authors have no conflict of interest.

\section{Received: 9 May 2011 Accepted: 26 July 2011 Published: 26 July 2011}

\section{References}

1. Modjtahedi $\mathrm{H}$, Essapen S: Epidermal growth factor receptor inhibitors in cancer treatment: advances, challenges and opportunities. Anticancer Drugs 2009, 20:851-855.

2. Lynch TJ, Bell DW, Sordella R, Gurubhagavatula S, Okimoto RA, Brannigan BW, Harris PL, Haserlat SM, Supko JG, Haluska FG, Louis DN, Christiani DC, Settleman J, Haber DA: Activating mutations in the epidermal growth factor receptor underlying responsiveness of nonsmall-cell lung cancer to gefitinib. N Engl J Med 2004, 350:2129-39.

3. Paez JG, Jänne PA, Lee JC, Tracy S, Greulich H, Gabriel S, Herman P, Kaye FJ, Lindeman N, Boggon TJ, Naoki K, Sasaki H, Fujii Y, Eck MJ, Sellers WR, Johnson BE, Meyerson M: EGFR mutations in lung cancer: correlation with clinical response to gefitinib therapy. Science 2004, 304:1497-1500.

4. Mok TS, Wu YL, Thongprasert S, Yang CH, Chu DT, Saijo N, Sunpaweravong $P$, Han B, Margono B, Ichinose $Y$, Nishiwaki $Y$, Ohe $Y$, Yang JJ, Chewaskulyong B, Jiang H, Duffield EL, Watkins CL, Armour AA, Fukuoka M: Gefitinib or Carboplatin-Paclitaxel in Pulmonary Adenocarcinoma. N Engl J Med 2009, 361:947-957.

5. Mitsudomi T, Morita S, Yatabe Y, Negoro S, Okamoto I, Tsurutani J, Seto T, Satouchi M, Tada H, Hirashima T, Asami K, Katakami N, Takada M, Yoshioka H, Shibata K, Kudoh S, Shimizu E, Saito H, Toyooka S, Nakagawa K, Fukuoka M, West Japan Oncology Group: Gefitinib versus cisplatin plus docetaxel in patients with non-small-cell lung cancer harboring mutations of the epidermal growth factor receptor (WJTOG3405): an open label, randomized phase 3 trial. Lancet Oncol 2010, 11:121-128.

6. Maemondo M, Inoue A, Kobayashi K, Sugawara S, Oizumi S, Isobe H, Gemma A, Harada M, Yoshizawa H, Kinoshita I, Fujita Y, Okinaga S, Hirano H, Yoshimori K, Harada T, Ogura T, Ando M, Miyazawa H, Tanaka T, Saijo Y, Hagiwara K, Morita S, Nukiwa T, North-East Japan Study Group: Gefitinib or chemotherapy for non-small-cell lung cancer with mutated EGFR. N Engl J Med 2010, 362:2380-2388.

7. Inoue A, Saijo Y, Maemondo M, Gomi K, Tokue Y, Kimura Y, Ebina M, Kikuchi T, Moriya T, Nukiwa T: Severe acute interstitial pneumonia and gefitinib. Lancet 2003, 361:137-139.

8. Inomata S, Takahashi H, Nagata M, Yamada G, Shiratori M, Tanaka H, Satoh M, Saitoh T, Sato T, Abe S: Acute lung injury as an adverse event of gefitinib. Anticancer Drugs 2004, 15:461-467.

9. Hotta K, Kiura K, Tabata M, Harita S, Gemba K, Yonei T, Bessho A, Maeda T, Moritaka T, Shibayama T, Matsuo K, Kato K, Kanehiro A, Tanimoto Y, Matsuo K, Ueoka H, Tanimoto M: Interstitial lung disease in Japanese patients with non-small cell lung cancer receiving gefitinib: an analysis of risk factors and treatment outcomes in Okayama Lung Cancer Study Group. Cancer J 2005, 11:417-424
10. Ando M, Okamoto I, Yamamoto N, Takeda K, Tamura K, Seto T, Ariyoshi Y, Fukuoka M: Predictive factors for interstitial lung disease, antitumor response, and survival in non-small-cell lung cancer patients treated with gefitinib. J Clin Oncol 2006, 24:2549-2556.

11. Kudoh S, Kato H, Nishiwaki Y, Fukuoka M, Nakata K, Ichinose Y, Tsuboi M, Yokota S, Nakagawa K, Suga M, Japan Thoracic Radiology Group, Jiang H, Itoh Y, Armour A, Watkins C, Higenbottam T, Nyberg F: Interstitial lung disease in Japanese patients with lung cancer: a cohort and nested case-control study. Am J Respir Crit Care Med 2008, 177:1348-1357.

12. Nakagawa M, Nishimura T, Teramukai S, Tada H, Tanaka F, Yanagihara K, Furuse K, Wada H, Fukushima M: Interstitial lung disease in gefitinibtreated Japanese patients with non-small cell lung cancer - a retrospective analysis: JMTO LC03-02. BMC Res Notes 2009, 2:157.

13. Hotta K, Kiura K, Takigawa N, Yoshioka H, Harita S, Kuyama S, Yonei T, Fujiwara K, Maeda T, Aoe K, Ueoka H, Kamei H, Umemura S, Moritaka T, Segawa Y, Kawai H, Bessho A, Kato K, Tabata M, Tanimoto M: Comparison of the incidence and pattern of interstitial lung disease during erlotinib and gefitinib treatment in Japanese Patients with non-small cell lung cancer: the Okayama Lung Cancer Study Group experience. J Thorac Oncol 2010, 5:179-184.

14. Kohno N, Akiyama M, Kyoizumi S, Hakoda M, Kobuke K, Yamakido M: Detection of soluble tumor-associated antigens in sera and effusions using novel monoclonal antibodies, $\mathrm{KL}-3$ and $\mathrm{KL}-6$, against lung adenocarcinoma. Jpn J Clin Oncol 1988, 18:203-216.

15. Kohno N, Inoue Y, Hamada H, Fujioka S, Fujino S, Yokoyama A, Hiwada K, Ueda N, Akiyama M: Difference in sero-diagnostic values among KL-6associated mucins classified as cluster 9. Int J Cancer 1994, 57(suppl 8):81-83.

16. Hirasawa Y, Kohno N, Yokoyama A, Inoue Y, Abe M, Hiwada K: KL-6, a human MUC1 mucin, is chemotactic for human fibroblasts. Am J Respir Cell Mol Biol 1997, 17:501-507.

17. Ohyabu N, Hinou H, Matsushita T, Izumi R, Shimizu H, Kawamoto K, Numata Y, Togame H, Takemoto H, Kondo H, Nishimura S: An Essential Epitope of Anti-MUC1 Monoclonal Antibody KL-6 Revealed by Focused Glycopeptide Library. J Am Chem Soc 2009, 131:17102-17109.

18. Kohno N, Kyoizumi S, Awaya Y, Fukuhara H, Yamakido M, Akiyama M: New serum indicator of interstitial pneumonitis activity. Sialylated carbohydrate antigen KL-6. Chest 1989, 96:68-73.

19. Kohno N, Hamada H, Fujioka S, Hiwada K, Yamakido M, Akiyama M: Circulating antigen $\mathrm{KL}-6$ and lactate dehydrogenase for monitoring irradiated patients with lung cancer. Chest 1992, 102:117-122.

20. Kohno N, Awaya Y, Oyama T, Yamakido M, Akiyama M, Inoue Y, Yokoyama A, Hamada H, Fujioka S, Hiwada K: KL-6, a mucin-like glycoprotein, in bronchoalveolar lavage fluid from patients with interstitial lung disease. Am Rev Respir Dis 1993, 148:637-642.

21. Yokoyama A, Kohno N, Hamada H, Sakatani M, Ueda E, Kondo K, Hirasawa Y, Hiwada K: Circulating KL-6 predicts the outcome of rapidly progressive idiopathic pulmonary fibrosis. Am J Respir Crit Care Med 1998, 158:1680-1684.

22. Kohno N: Serum marker KL-6/MUC1 for the diagnosis and management of interstitial pneumonitis. J Med Invest 1999, 46:151-158.

23. Ohnishi H, Yokoyama A, Kondo K, Hamada H, Abe M, Nishimura K, Hiwada K, Kohno N: Comparative study of KL-6, surfactant protein-A, surfactant protein- $D$, and monocyte chemoattractant protein-1 as serum markers for interstitial lung diseases. Am J Respir Crit Care Med 2002, 165:378-381.

24. Yokoyama A, Kondo K, Nakajima M, Matsushima T, Takahashi T, Nishimura M, Bando M, Sugiyama Y, Totani Y, Ishizaki T, Ichiyasu H, Suga M, Hamada H, Kohno N: Prognostic value of circulating KL-6 in idiopathic pulmonary fibrosis. Respirology 2006, 11:164-168.

25. Nakashima T, Yokoyama A, Ohnishi H, Hamada H, Ishikawa N, Haruta Y, Hattori N, Tanigawa K, Kohno N: Circulating KL-6/MUC1 as an independent predictor for disseminated intravascular coagulation in acute respiratory distress syndrome. J Intern Med 2008, 263:432-439.

26. Ohshimo S, Bonella F, Grammann N, Starke K, Cui A, Bauer PC, Teschler H, Kohno N, Guzman J, Costabel U: Serum KL-6 as a novel disease marker in adolescent and adult cystic fibrosis. Sarcoidosis Vasc Diffuse Lung Dis 2009, 26:47-53.

27. Ohnishi H, Yokoyama A, Yasuhara Y, Watanabe A, Naka T, Hamada H, Abe M, Nishimura K, Higaki J, Ikezoe J, Kohno N: Circulating KL-6 levels in patients with drug induced pneumonitis. Thorax 2003, 58:872-875. 
28. Inata J, Hattori N, Yokoyama A, Ohshimo S, Doi M, Ishikawa N, Hamada H, Kohno N: Circulating KL-6/MUC1 mucin carrying sialyl Lewis ${ }^{a}$ oligosaccharide is an independent prognostic factor in patients with lung adenocarcinoma. Int J Cancer 2007, 120:2643-2649.

29. Tanaka S, Hattori N, Ishikawa N, Shoda H, Takano A, Nishino R, Okada M, Arihiro K, Inai K, Hamada H, Yokoyama A, Kohno N: Krebs von den Lungen-6 (KL-6) is a prognostic biomarker in patients with surgically resected non-small cell lung cancer. Int J Cancer 2011.

30. Ishikawa N, Hattori N, Yokoyama A, Tanaka S, Nishino R, Yoshioka K, Ohshimo S, Fujitaka K, Ohnishi H, Hamada H, Arihiro K, Kohno N: Usefulness of monitoring the circulating Krebs von den Lungen-6 levels to predict the clinical outcome of patients with advanced non-small cell lung cancer treated with epidermal growth factor receptor tyrosine kinase inhibitors. Int J Cancer 2008, 122:2612-2620.

31. American Thoracic Society: Idiopathic pulmonary fibrosis: diagnosis and treatment. International consensus statement. American Thoracic Society (ATS), and the European Respiratory Society (ERS). Am J Respir Crit Care Med 2000, 161:646-664.

32. American Thoracic Society, European Respiratory Society: American Thoracic Society/European Respiratory Society International Multidisciplinary Consensus Classification of the Idiopathic Interstitial Pneumonias. This joint statement of the American Thoracic Society (ATS), and the European Respiratory Society (ERS) was adopted by the ATS board of directors, June 2001 and by the ERS Executive Committee, June 2001. Am J Respir Crit Care Med 2002, 165:277-304.

33. Webb WR, Müller NL, Naidich DP: HIGH-RESOLUTION CT of the LUNG. 4 edition. Philadelphia: Lippincott Williams \& Wilkins; 2009.

34. Goddard PR, Nicholson EM, Laszlo G, Watt I: Computed tomography in pulmonary emphysema. Clin Radiol 1992, 33:379-387.

35. Müller NL, White DA, Jiang H, Gemma A: Diagnosis and management of drug-associated interstitial lung disease. Br J Cancer 2004, 91(Suppl 2): S24-30.

36. Endo M, Johkoh T, Kimura K, Yamamoto N: Imaging of gefitinib-related interstitial lung disease: multi-institutional analysis by the West Japan Thoracic Oncology Group. Lung Cancer 2006, 52:135-140.

37. Nagai Y, Miyazawa H, Tanaka T, Udagawa K, Kato M, Fukuyama S, Yokote A, Kobayashi K, Kanazawa M, Hagiwara K: Genetic heterogeneity of the epidermal growth factor receptor in non-small cell lung cancer cell lines revealed by a rapid and sensitive detection system, the peptide nucleic acid-locked nucleic acid PCR clamp. Cancer Res 2005, 65:7276-7282.

38. Camus $P$, Kudoh $S$, Ebina M: Interstitial lung disease associated with drug therapy. Br J Cancer 2004, 91:S18-23.

39. Armour A: Gefitinib in advanced non-small cell lung cancer: clinical experience in patients of Asian origin. Asia Pac J Clin Oncol 2007, 3:66-78.

40. Inoue Y, Barker E, Daniloff E, Kohno N, Hiwada K, Newman LS: Pulmonary epithelial cell injury and alveolar-capillary permeability in berylliosis. Am J Respir Crit Care Med 1997, 156:109-115.

41. Kitajima H, Takahashi H, Harada K, Kanai A, Inomata S, Taniguchi H, Saikai T, Abe S: Gefitinib-induced interstitial lung disease showing improvement after cessation: disassociation of serum markers. Respirology 2006, 11:217-220.

doi:10.1186/1465-9921-12-97

Cite this article as: Kawase et al:: Change in serum KL-6 level from baseline is useful for predicting life-threatening EGFR-TKIs induced interstitial lung disease. Respiratory Research 2011 12:97.

\section{Submit your next manuscript to BioMed Central and take full advantage of:}

- Convenient online submission

- Thorough peer review

- No space constraints or color figure charges

- Immediate publication on acceptance

- Inclusion in PubMed, CAS, Scopus and Google Scholar

- Research which is freely available for redistribution

Submit your manuscript at www.biomedcentral.com/submit 